\title{
Matlab-Based
}

\section{Design and Implementation of Time-Frequency Analyzer}

\author{
Abdul-Bary Raouf Sulaiman \\ Soad Taha Abed \\ College of Electronics Eng. \\ Technical Institute
}

\begin{abstract}
Spectrum analysis uses Fourier analysis for detecting the spectrum of a signal. The signals to be analyzed must be stationary that their spectra must not varying with time. For signals whose spectra varying with time (non-stationary) such as seismic signal, biomedical signal, speech signal, Joint Time-Frequency Analysis (JTFA) must be used. The work is to design PC- based Joint Time- Frequency Analyzer including the methods that used to calculate and display energy in Time-Frequency domain. These methods are the Short time Fourier transforms (STFT), Wavelet Analysis (WT), and Wigner-Ville distribution (WVD). Using the Graphical User Interface (GUI) for the Matlab software, front panel is designed as control panel involving all functions and tools necessary for the analysis.
\end{abstract}

Hardware requirements for interfacing the signal to the PC are considered and designed. The sound card used for signals in the audio range. External signals can be recorded, displayed, measured, stored and processed in time-domain, frequency-domain, and time-frequency domain.

Keywords: Digital signal processing, JTFA, Time-Frequency Analyzer
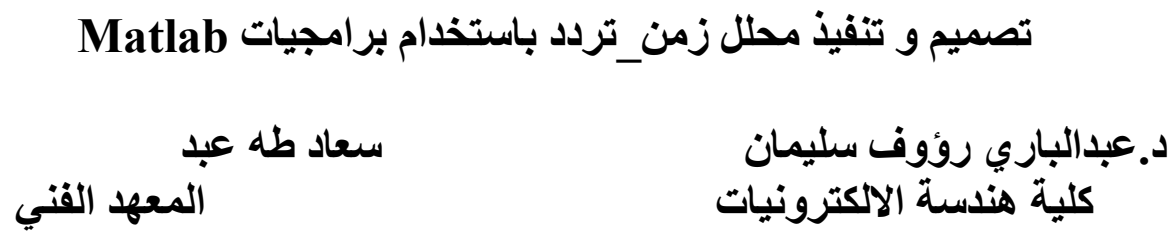

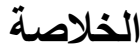

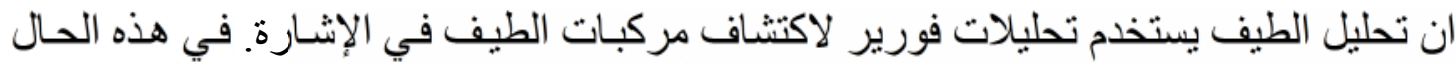
يجب ان تكون الإشـار ات المر ا د تحليلها مستثرة أي ان طيفها لايتغير مـع الزمن. أمـا بالنسبة الطية 


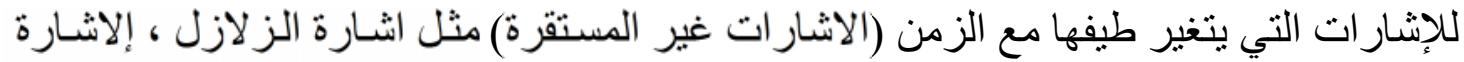

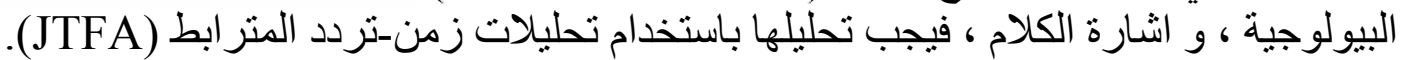

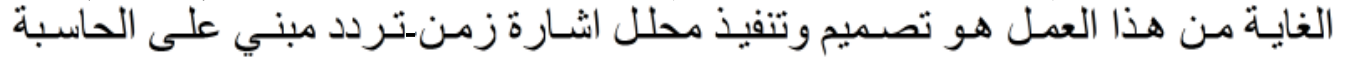

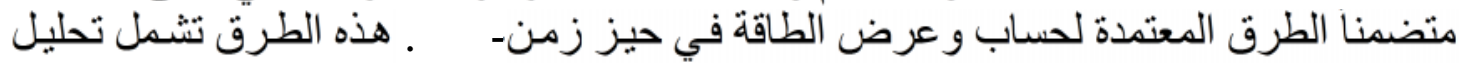

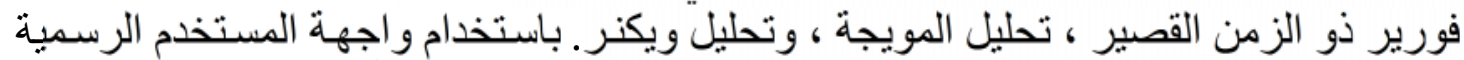

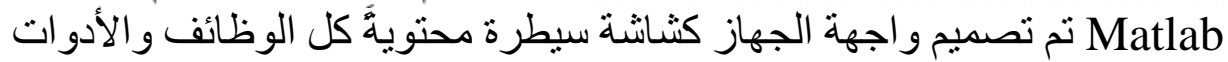

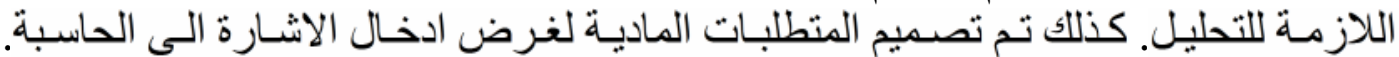
استخدام بطاقة الصوت كلادخال الاشار ات النهات السمعية.

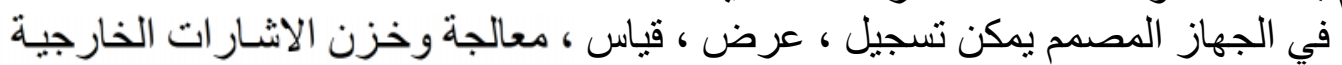
في حيز الزمن ، حيز التردد ، وحيز زمن-

I. Introduction

Receinqde 17 Fourter 2 Ppannsform is not always the Accepted 3 tSepnalyze real life signals, which are usually of finite or perhaps even relatively short duration. Common example includes speech signal. Despite the fact that the frequency content of most signals changes with time, the classical Fourier theory only allows to analyze a signal in the time domain or in the frequency domain. To over come this problem many alternatives such as time dependent spectrum has been developed and widely studied; these new technique is known as Joint Time Frequency Analysis (JTFA) [1].
\end{abstract}

\title{
1.1 Literature Review
}

The development of Joint Time-Frequency Analysis began more than half of century ago. It is the most straightforward approach of characterizing signal's frequency as a

function of time.

Chester, F.J. Taylor and M. Doyl [2] were the first to apply the Wigner-Ville distribution for the analysis of speech signal[1984]. ]. L.Cohen [3] gives a review for a joint function of time and frequency that describe the energy density of a signal simultaneously in time and frequency [1989].

The National Instruments produced Joint Time-Frequency Analysis Tool Kit using Labview ${ }^{\mathrm{TM}}$ software[4]. Anna H. Pryor, Marianne Moher [5] use the Short time Fourier transform and Continuous Wavelet transform to overcome the shortcoming of traditional spectral analysis 2001. 


\section{I.2 Aim and structure}

The aim of the research is to design and implement Joint TimeFrequency Analyzer, Including the Joint Time-Frequency Analysis family that are the Short Time Fourier Transform (STFT), Wavelet Transform (WT), and Wigner-Ville Distribution (WVD). These methods are applied to speech signal, which is a good example of non-stationary signals

The paper falls into five sections. After this introduction, a basic theory for the Joint Time-Frequency analysis tools is presented in section two. Section three explains the design of the Time-Frequency analyzer. Results and description of speech and other signals and their representations is presented in section four. Conclusions are introduced in section five.

\section{Theory \\ I1.1 Joint Time-Frequency Analysis (JTFA)}

In joint time-frequency analysis the time and frequency domain representations of a signal are combined into a time-frequency energy density function. This time-frequency representation can be used to study change of frequency content of a signal with time. There are number of possible time-frequency representations such as:

1-Short Time Fourier Transform (STFT)

2-Wavelet Transform (WT).

3- Wigner-Ville distribution (WVD).

\section{I1.2 Short Time Fourier Transform (STFT)}

In signal analysis one often encounters the so-called short-time Fourier transform, or windowed Fourier transform. The mathematical definition of the short time Fourier transforms is given in equation (1) 


$$
\operatorname{STFT}(t, w)=\int x(\tau) h^{*}(t-\tau) e^{-j \omega \tau} d \tau
$$

Where $\mathrm{x}(\mathrm{t})$ is the signal to be analyzed, $\mathrm{h}(\mathrm{t})$ a window function around the time $\tau$ [6]. Because the window function $h(t)$ has a short duration, the Fourier transform of $x(t) . h(t)$ reflects the frequency properties of the signal. During the analysis the window function is shifted in time over the entire signal and consecutive overlapped fast Fourier transformations (FFT) are performed and finally a time frequency representation is obtained. The size of the window determines the time accuracy, smaller window the better time resolution, poor frequency resolution and vice versa.

The Fourier spectrogram is the technique currently being used for the time frequency analysis and it is found from STFT, it gives the time frequency distribution of the energy in the signal [7]

$$
F S(t, w)=\left.\int x(\tau) h^{*}(t-\tau) e^{-j \omega \tau} d \tau\right|^{2}
$$

\section{I1.3 Continuous Wavelet Analysis(CWT)}

The Continuous Wavelet Transform (CWT) is a time frequency decomposition, which links a time (or space) domain function to its timescale wavelet domain representation. The concept of scale is broadly related to frequency. Small scales relate to short duration, high frequency features and correspondingly, large scales relate to long duration, low frequency features. The Continuous Wavelet Transform compares the signal with dilated and time shifted versions of a signal basis function called the mother wavelet [8]. The definition of CWT is given by::

$$
w(a, b)=\frac{1}{\sqrt{a}} \int x(t) \psi *\left[\frac{t-b}{a}\right] d t
$$


Where $\mathrm{x}(\mathrm{t})$ is any signal with finite energy, $\psi(\mathrm{t})$ is the mother wavelet, a is the dilation (or scale) of the mother wavelet and $b$ is the time-shift (or translation) of the wavelet with respect to the signal. If $\psi(\mathrm{t})$ is considered as the impulse response of a band pass filter, the CWT can be interpreted as band pass analysis. As the scale factor a is increased, the center frequency and bandwidth of the band pass filter will increase.

For a fixed scale, equation (3) then becomes a convolution with the scaled and time-revered wavelet:

$W(t, a)=\frac{1}{\sqrt{|a|}} X(t) * \Psi_{a}(t), \quad \psi_{a}(t)=\psi *\left(-\frac{t}{a}\right)$

The factor $\mid \mathrm{a}^{-1 / 2}$ is included to ensure that all the scaled wavelet functions have the same energy.

As the square of STFT is Fourier Spectrogram (FS), the square of continuous wavelet transform is the Scalogram, which can be used to describe the energy distribution of a signal in the time-frequency domain.

$$
w(a, b)=\left|\int_{-\infty}^{\infty} x(t) \psi *\left[\frac{t-b}{a}\right] d t\right|^{2}
$$

\section{I1.4 Wigner Ville Distribution(WVD)}

The Wigner-Ville distribution (WVD) is the most popular representative of the time-frequency distributions (TFD) because it is simple and powerful. A time-frequency distribution is a transform that maps a 1-D signal into a 2-D time-frequency map, which describes the spectral content of the data evolves with time [9]. A Hungarian-born American physicist, Eugene P. Wigner, originally developed the WVD in the context of quantum mechanics. For a time signal $x(t)$ the Wigner_Ville distribution is given by[10]:

$$
W V D_{(t, \omega)}=\int_{-\infty}^{\infty} x(t-\tau / 2) \cdot x *(t+\tau / 2) e^{-j \omega \tau} d \tau
$$

According to equation (6) the Winger Ville distribution can be considered as the Fourier transform of the auto-correlation function of the 
signal $\mathrm{x}(\mathrm{t})$. Compared to the short time Fourier transform (STFT) for the calculation of the WVD no analysis window function $h(t)$ is needed, and the WVD does not suffer from the window effects. The problem of the WVD is the so called cross- term interference. Because this term reflects the correlation of two signal components, it is named cross term. Many researchers have developed alternative kernels to overcome this problem. On of the very popular kernel distributions is the Choi-William [10 ].

\section{Joint Time-Frequency Analyzer Design}

Figure(1) shows the front panel of the joint time-frequency analyzer including all figure places and control icons requiring for displaying, read, measure and manipulate the results of the signal analysis in time, frequency, and time-frequency domains.

GUI Toolbox, Data Acquisition Toolbox, Signal processing Toolbox, and Higher Order Spectral Analysis Toolbox operating under Matlab v6.5 software[11] were used to design the front panel, acquire, process, and analyze the signal.

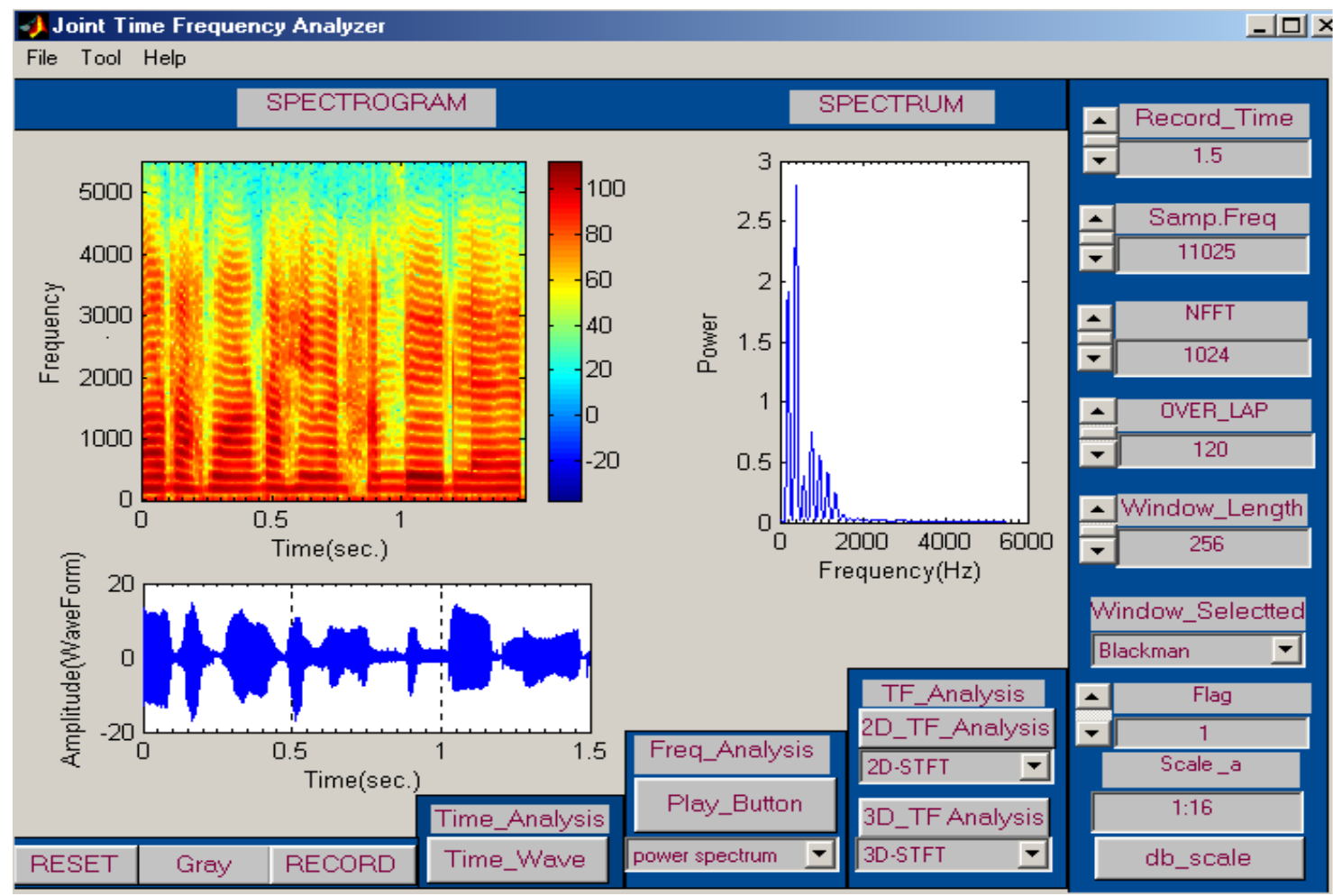

Fig (1) Joint Time Frequency Analyzer based on Matlab 
The following sections explain how to manipulate controls and read indicators in the Joint Time-Frequency Analyzer.

\section{- Recording:}

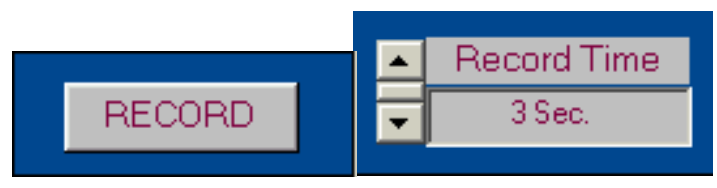

It is initiated through the matlab graphical user interface (GUI) by clicking on the record button. The duration of the recording can be adjusted to be any where from .03 to 3 second. The duration can be modified to record for longer duration if desired. Upon being clicked, the record button executes a function that reads in mono data from the microphone or function generator on the sound card and stores it as a mat lab vector.

- Setting The Sampling Frequency

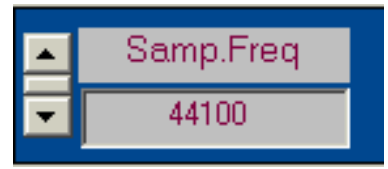

The samp. Freq control is used to specify the sampling frequency. While the sampling frequency does not affect the computation results, it does affect the short time Fourier transform display.

\subsection{Data Display}

The joint time-frequency analyzer uses the following data display:

- Frequency Analysis

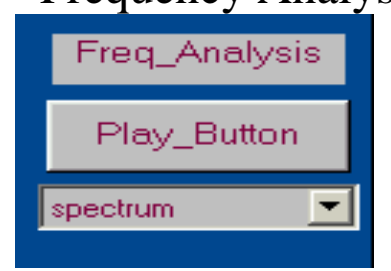

The Spectrum control shows either power spectrum or instantaneous spectrum, depending on the setting that has been chosen.

- The Time-Analysis: 
The Time- Wave shows the time waveform of the analyzed signal.

- TF-Analysis

\begin{tabular}{l} 
TF_Analysis \\
\hline 2D_TF_Analysis \\
\hline 2D-STFT \\
\hline 2D_STFT \\
2D_WIG2C \\
2D_WAVELET \\
\hline 3D_TF_Analysis \\
\hline 3D-STFT \\
\hline
\end{tabular}

The TF-Analysis shows the time-dependent spectrum for the chosen analysis method in two dimensions and three dimensions.

3.3 Selecting Joint Time-Frequency Analysis (JTFA) Method

With the joint time frequency analyzer one of the following analysis methods can be chosen

- STFT Spectrogram

- Wavelet transform

- Wigner-Ville distribution

TF-Analysis control is used to select the analysis method. After choosing the analysis method, clicking 2D-TF-Analysis button or 3D-TFAnalysis to compute the STFT, CWT, and WVD in 2D or 3D. The following sections provide more information about each of the joint time frequency analysis methods in the signal processing.

\section{STFT Based Spectrogram}




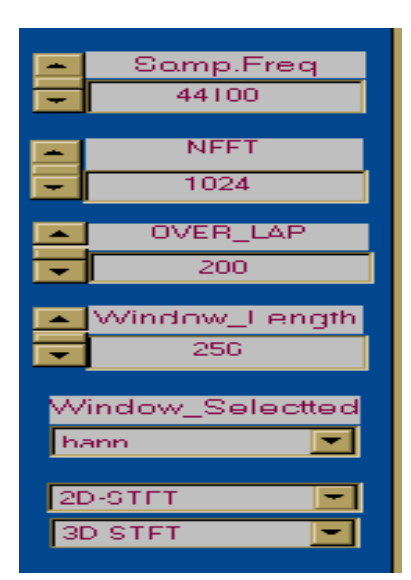

The frequency content of any signal as a function of time can be calculated using spectrogram function. This method is computed using the matlab's built in "specgram" command [11].

A Gaussian signal borrowed from a Matlab demonstration to the analyzer is shown in Figure (2). The upper left plot shows the STFT spectrogram, which shows the frequency contents as a function of time. Frequency is on the vertical axis and time on the horizontal axis. Brown color indicates frequency with higher energy, while blue color indicates frequency with smaller energy. The beauty of the spectrogram is that clearly illustrates how the frequency of a signal varies with time. The bottom plot shows the time waveform, The upper right plot shows the power as a function of frequency.

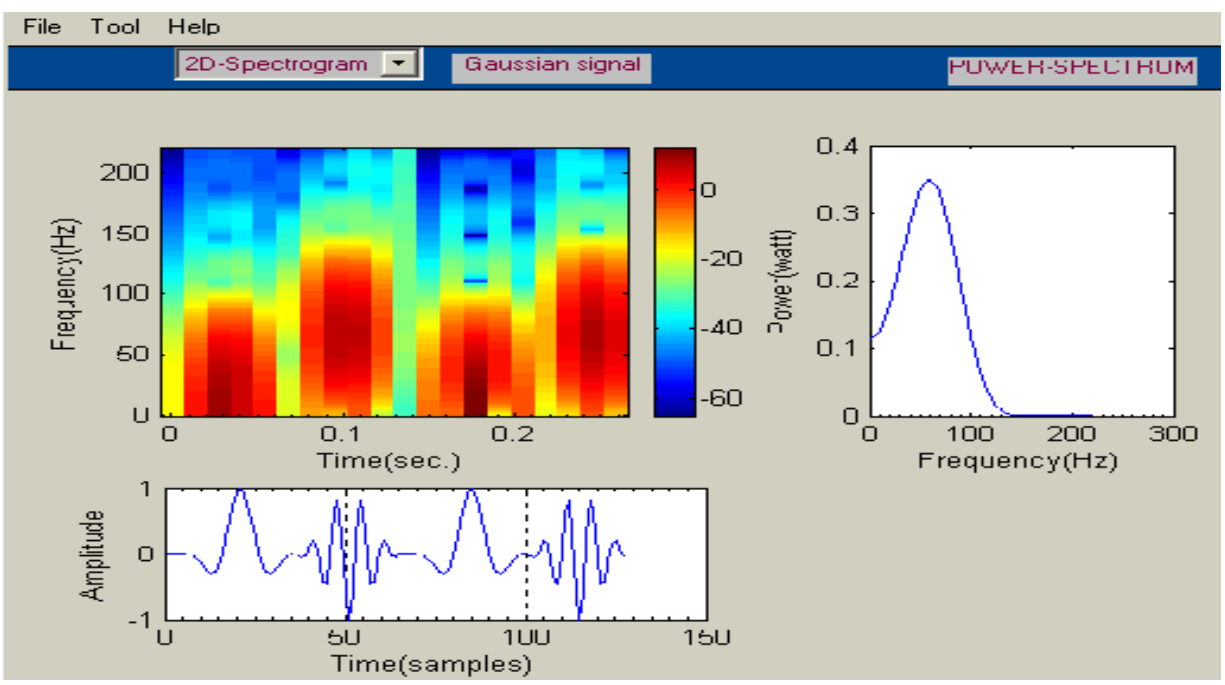

Fig (2) the STFT, Power Spectrum and Time waveform of a Gaussian signal 


\section{The Continuous Wavelet Transform:}

The second method of joint time-frequency analysis is the wavelet transform. The wavelet transform, is a time frequency method that builds on the idea of the STFT whereas the STFT is limited in resolution because of its fixed window size, the WT use variable window size, with short windows for high frequencies and long windows for low frequencies. The wavelet transform is the sum over all time of the signal multiplied by scaled, shifted versions of the wavelet. This process produces wavelet coefficients that are a function of scale and position. Continuous wavelet coefficients of the vector $\mathrm{x}$ at real, positive scales is given as [11]:

$$
\text { C= cwt (x, a,'sym4','Abs1v1', b, plot) }
$$

C continuous wavelet coefficient,

$\mathrm{x} \quad$ The signal to be analyzed.

a Scale (or dilation) of the mother wavelet, 
b The time-shift or (translation) of the wavelet with respect to the signal, Sym4 mother wavelet transform name,

Abs1v1 (Absolute value and By scale).

Choosing the (2D-Wavelet) from the control of the Joint TimeFrequency Analyzer, clicking the (2D-TF-Analysis) button, the Gaussian signal is analyzed using the Continuous Wavelet analysis which is illustrated in the upper left plot of Figure (3), which shows the scale contents as a function of time.

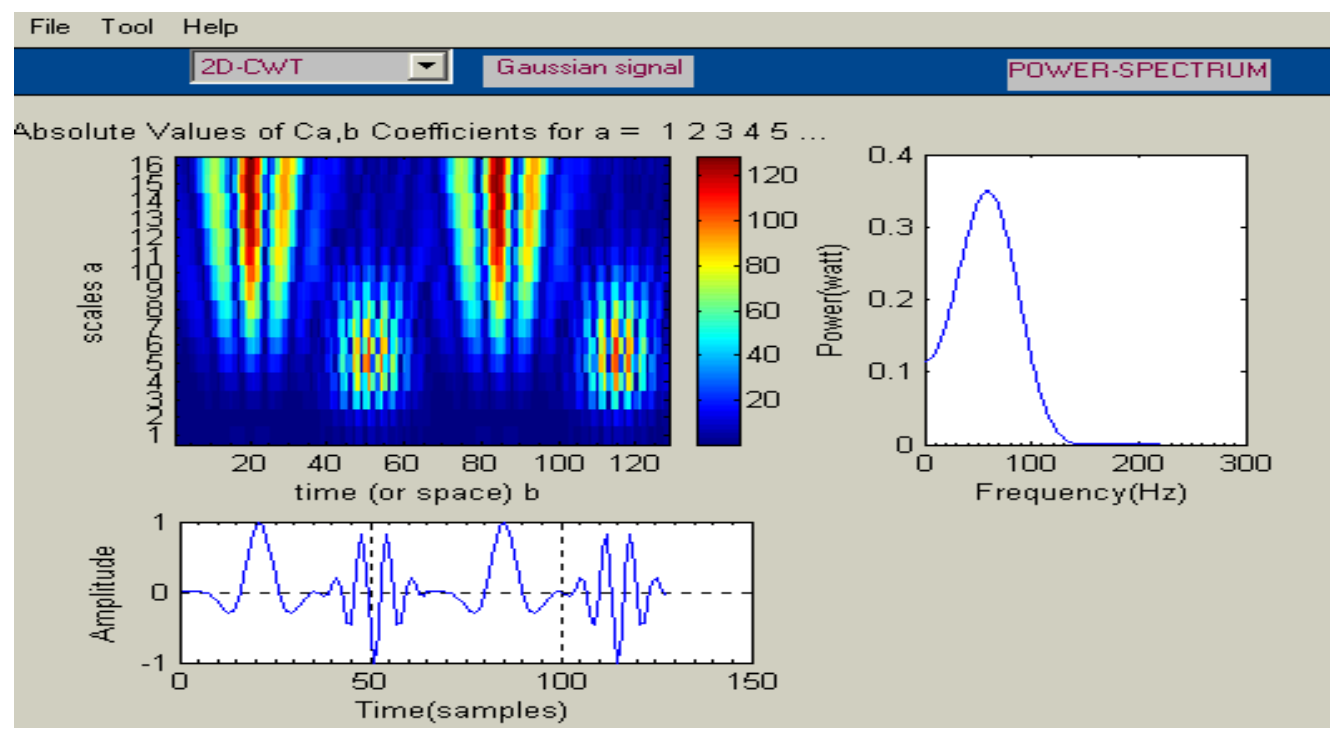

Fig (3) the CWT, Power Spectrum and Time waveform of a Gaussian signal 
* Wigner-Ville Distribution: The Wigner-Ville distribution (WVD) with Choi-William filtering provides high time-frequency resolution. However, it may suffer from serious cross-term interference if the analyzed signal consists of multiple components. The Wigner-Ville distribution is computed using higher order spectral analysis Toolbox of matlab version 5, uses the "wig2c" Matlab command [11].

Choosing the (2D-WIG2C) from the control of the Joint Time-Frequency Analyzer, clicking the (2D-TF-Analysis) button, the Gaussian signal is analyzed using the Wigner-Ville Distribution, which is illustrated in the upper left plot of Figure (4).

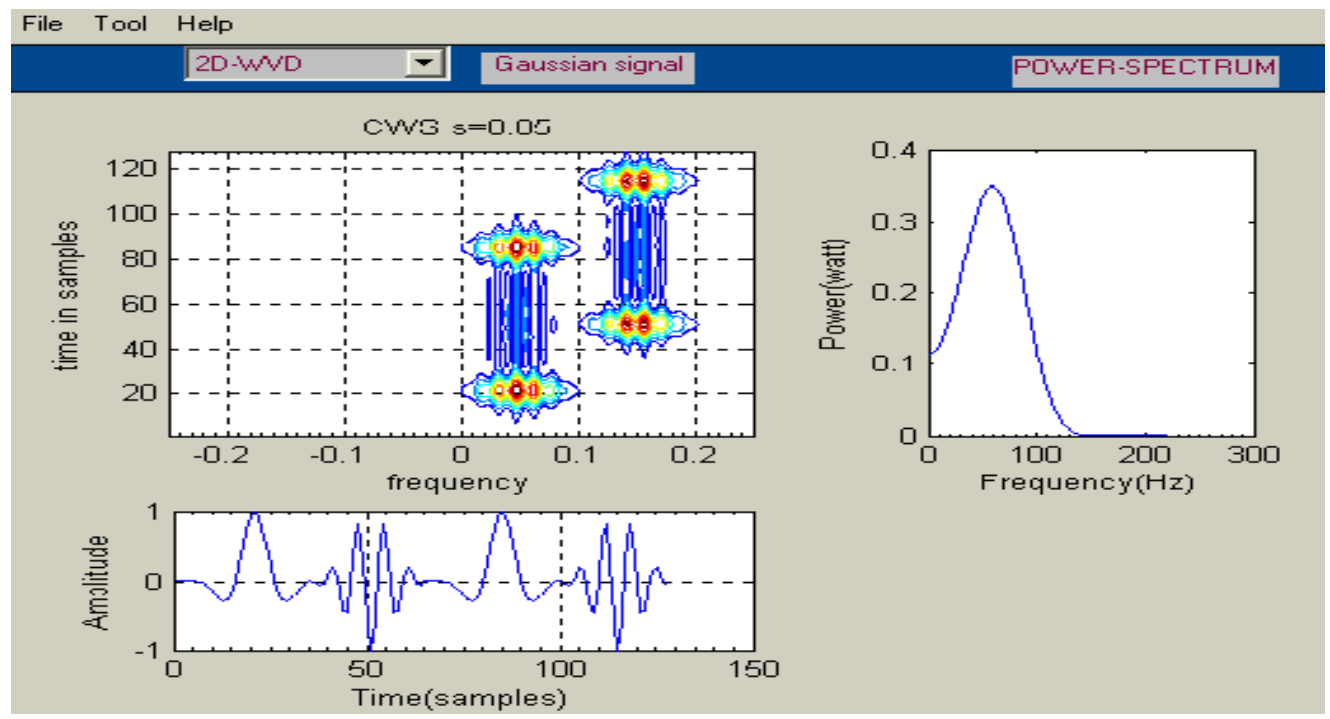

Fig (4) the WVD, Power Spectrum and Time waveform of a Gaussian signal 


\subsection{Matlab recording \& Analysis Program:}

Here a matlab program is presented. The program is intended to simplify the recording and basic editing of any waveform such as speech waveform as well as to present the Short Time Fourier Transform spectrogram, Continuous Wavelet Transform (CWT), Wigner-Ville Distribution (WVD) and spectrum in a side-by-side format for ease of analysis.

\section{Results}

Speech waveforms will be analyzed here, and various features in the waveforms, spectrogram, and wavelet transform, Wigner-Ville distribution will be noted. The most prominent features in the spectrogram are the brown horizontal bands, called formants, corresponding to the frequencies of higher energy. In addition the spectrum of small segments of the waveform will be analyzed. This 2D spectrum will help to compare formant frequencies from sound to sound. The word "already" contains several different sounds, all of them voiced. Notice how the formants change with time. One significant feature that is also easy to identify in the spectrogram is the /d/ sound. This sound is called a stop, and for obvious reasons. When the $/ \mathrm{d} /$ is pronounced, the tongue temporarily stops air from leaving the oral cavity. This leads to small amplitude in the waveform and a 'hole' in the spectrogram. As illustrated in the upper left plot of Figure (5), the bottom plot is the time waveform, shows how the magnitude changes with time. The upper right plot is the power spectrum, shows the approximate locations of the formants.. The brown color indicates the frequencies of higher energy.

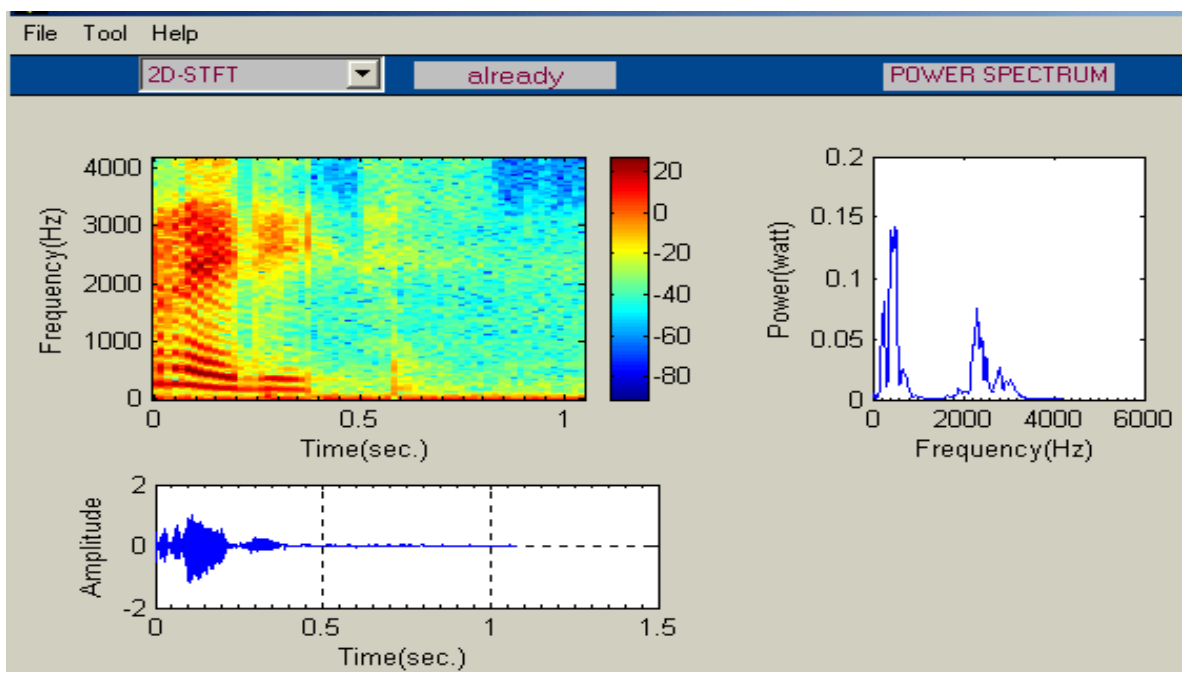


Choosing (2D-Wavelet) from the control of the Joint Time-Frequency Analyzer and clicking on the (2D-TF-Analysis) button, the word "already" is analyzed using the Continuous Wavelet Transform (CWT), which is illustrated in the upper left plot of Figure (6).

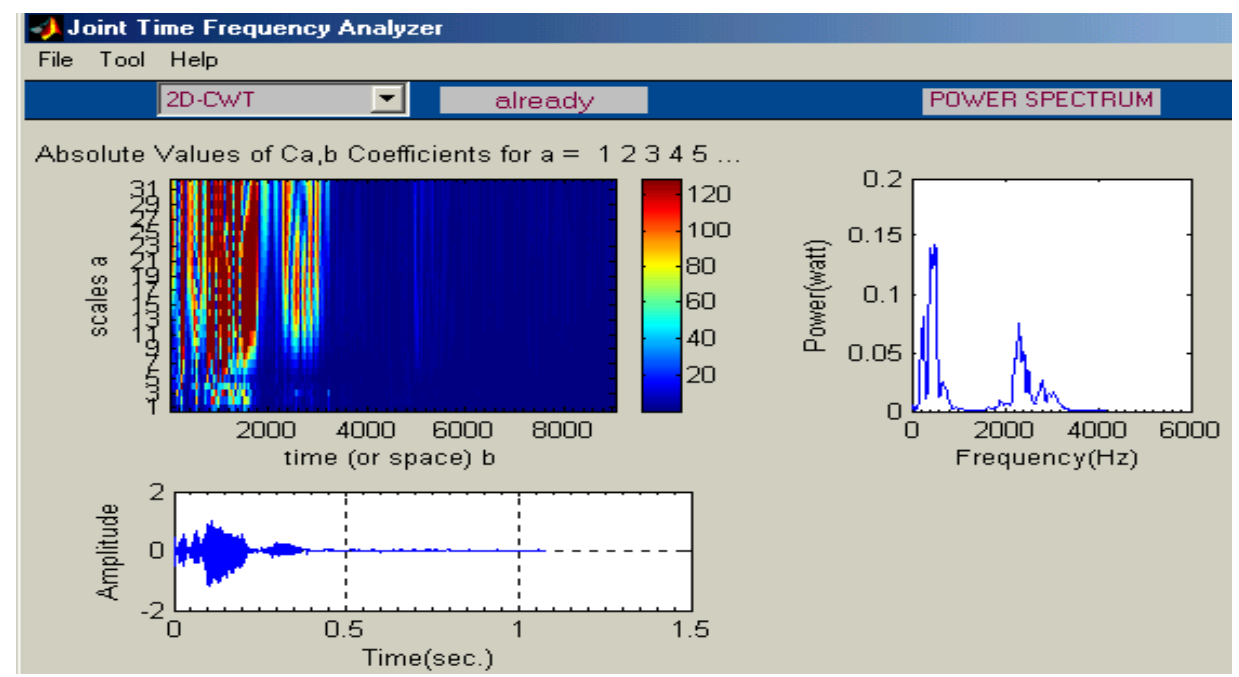

Fig (6) The CWT, time waveform, 
Choosing the (2D-WIG2C) from the control of the Joint Time-Frequency Analyzer and clicking on the (2D-TF-Analysis) button, the word "already" is analyzed using Wigner-Ville distribution, which is illustrated in the upper left plot of Figure (7).

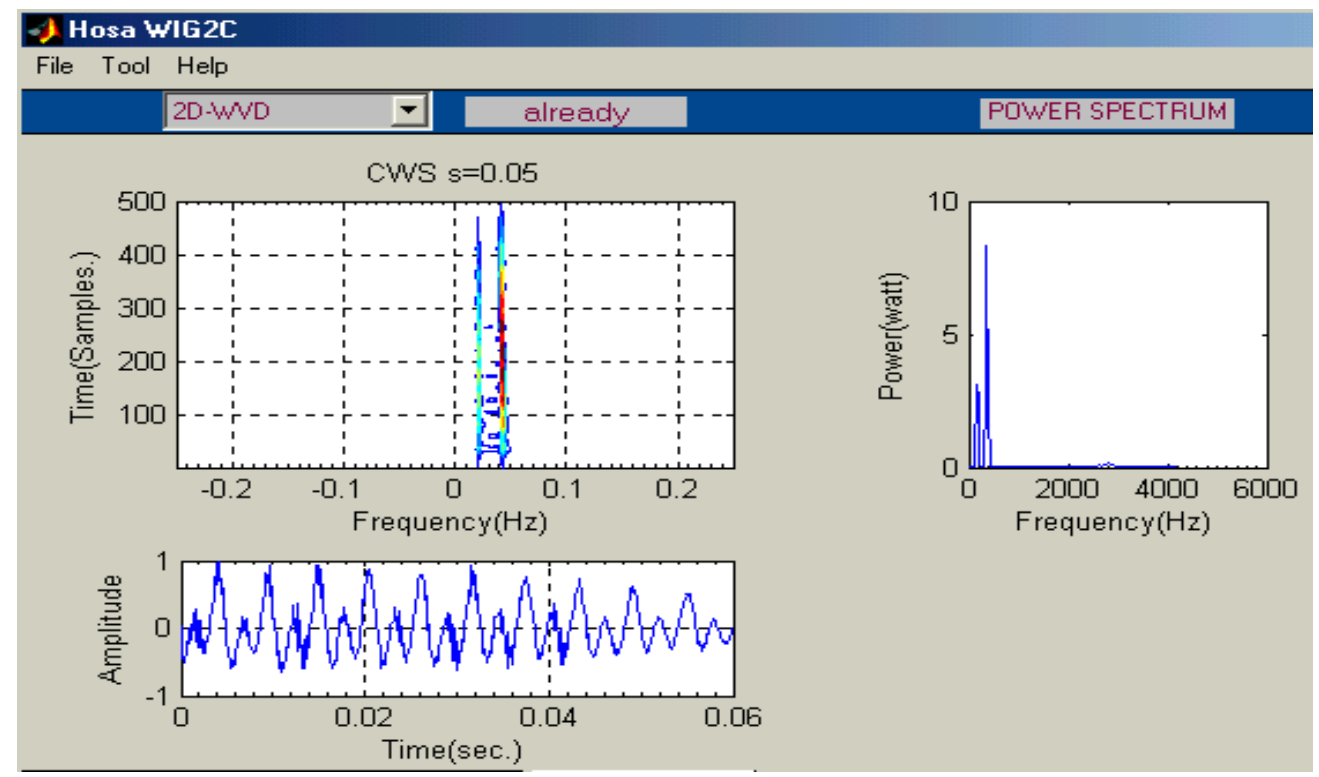

Fig (7) the WVD, time waveform, and Power Spectrum

of the word " already" 


\section{Signal energy in the joint time-frequency domain:}

The signal energy can be represented in three dimensions (3D) as illustrated in Figure (8). The upper left plot of this Figure shows the

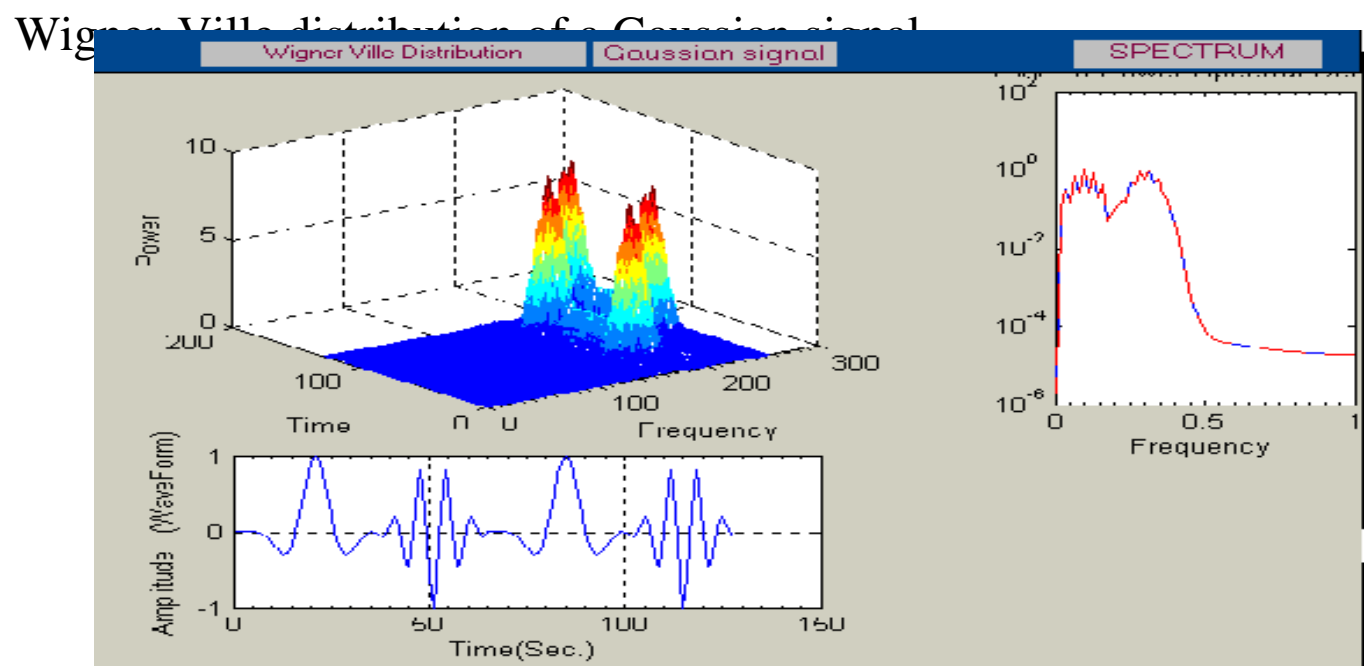

Fig (8) the time waveform, spectrum and WVD of a Gaussian signal in 3D 


\section{Conclusions}

Fourier transform is used to analyze stationary signals, that their spectra varying with time. For signals whose spectra varying with time (non-stationary), such as speech signals, joint time frequency analysis must be used. Joint Time-Frequency Analysis methods are the Short time Fourier transform (STFT), Wavelet transform (WT) and Wigner-Ville distribution (WVD). In this work joint time-frequency analyzer was designed and implemented including the above methods that used to calculate and display energy in time-frequency domain.

A visual screen is designed involving all tools needed to display, measure and analyze the signals in the time-frequency plane. The Matlab software has been chosen for its platform independence and graphical user interface (GUI) capabilities.

The Matlab swing GUI was used to facilitate the interpretation through graphical displays and parametric controls of various signalprocessing coefficient. The joint time-frequency analyzer designed has many powerful capabilities.

The data can be displayed as

- Linear or in decibel scale.

- Two dimensions or three dimension.

- Color or gray.

In addition to the above capabilities the joint time-frequency analyzer includes:

1-File icon contains five options, open, close, save as, print, print preview

2-Tool icon contains zoom on. 
3-Help icon contains help spectrogram demo, help general, help about CB.

The Joint Time-Frequency analyzer is used in analyzing signals from sound files or real time signals recorded from outside word. This PCbased device designed and implemented in this work is a powerful and helpful analysis tool in signal processing field.

\section{$\underline{\text { References }}$}

[1] Qian and Dapang Chen, "Understanding the Nature of Signals Whose Power Spectrum Change with Time. Joint Analysis," IEEE Signal Processing Magazine, 1999.

[2] D. Chester, F.J. Taylor, and M. Doyle, “ The Wigner Distribution, In speech processing applications'," J. Franklin Inst., Vol. 318, pp. 415-420, 1984.

[3] L. Cohen “ Time-Frequency Distributions-A review," (invited Paper). Proceeding of the IEEE, Vol.77, and No.7.July 1989

[4] National Instruments, LabVIEW ${ }^{\mathrm{TM}}$, Joint Time-Frequency Analysis Toolkit Reference Manual. November 1997 Edition, Part Number 320544C-01.

[5] Anna H.Pryor, Marianne Mosher, NASA Ames Research Center and David G.Lewicki, NASA Glenn Research Center,"The Application of Time-Frequency Methods to HUMS. Copyright By the American - Helicopter Society. May 9-11, 2001.

[6] Ingrid Daubechies, "The Wavelet Transform, Time-Frequency Localization and Signal," IEEE transactions. information theory, vol, 36,no.5, 1990.

[7] Flemming Pedersen, " Joint Time Frequency Analysis in Digital Signal Processing," Ph.D. Thesis, Department of Communication Technology, Institute of Electronic Systems, Aalborg University, Denmark, 2000 
[8] Thomas A. Ridsdil-Simth, “ The Application of The Wavelet To The Processing of Aeromagnetic Data," The University of Western Australia, Department of Geology and Geophysics, September 2000.

[9] Higher-Order Spectral Analysis Toolbox for Use With Matlab, MAthWorks Inc, $\quad$ MA, USA, 1998.

[10] Sulaiman,A.R., "Joint Time-Frequency analysis and its applications for non- $\quad$ stationary signals", Ph.D. Thesis, Elect. Eng. Dept., Univ. of Mosul, Jan. 2001

[11] The MathWorks, Inc. "Matlab the Language of Technical Computing", Version 6.5,2002,MA,USA. 